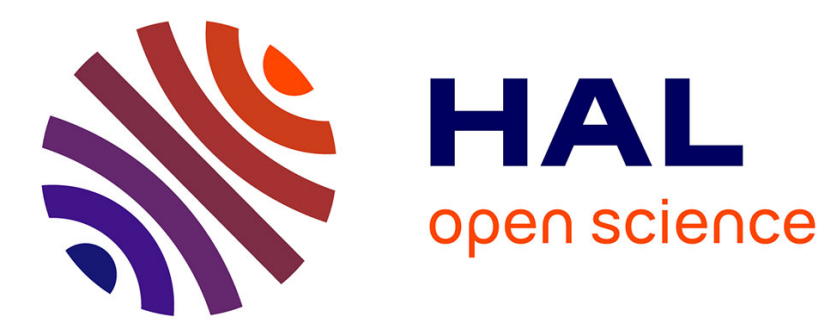

\title{
The Retrospective and Present Judgment of the Passage of Time in the Elderly
}

Sylvie Droit-Volet, Natalia Martinelli, Michaël Dambrun, Guillaume T. Vallet, Fanny Lorandi

\section{- To cite this version:}

Sylvie Droit-Volet, Natalia Martinelli, Michaël Dambrun, Guillaume T. Vallet, Fanny Lorandi. The Retrospective and Present Judgment of the Passage of Time in the Elderly. Timing \& Time Perception Reviews, 2021, 9 (4), pp.335-352. 10.1163/22134468-bja10031 . hal-03395980

\section{HAL Id: hal-03395980 \\ https://hal.science/hal-03395980}

Submitted on 13 Dec 2021

HAL is a multi-disciplinary open access archive for the deposit and dissemination of scientific research documents, whether they are published or not. The documents may come from teaching and research institutions in France or abroad, or from public or private research centers.
L'archive ouverte pluridisciplinaire HAL, est destinée au dépôt et à la diffusion de documents scientifiques de niveau recherche, publiés ou non, émanant des établissements d'enseignement et de recherche français ou étrangers, des laboratoires publics ou privés. 
Running head: Judgment of the passage of time in the elderly

The retrospective and present judgment of the passage of time in the elderly

Droit-Volet $^{1}$, S., Martinelli ${ }^{1}$, N., Dambrun ${ }^{1}$, M., Vallet ${ }^{1}$, G.T., \& Lorandi $^{2}$, F.

${ }^{1}$ Université Clermont Auvergne, CNRS, LAPSCO, F-63000 CLERMONT-FERRAND, FRANCE

${ }^{2}$ Nursing home, EHPAD, CUSSET, FRANCE

Correspondence concerning this article should be sent to Sylvie Droit-Volet, Université

Clermont Auvergne, Laboratoire de Psychologie Sociale et Cognitive (LAPSCO), CNRS, UMR 6024, 34 avenue Carnot, 63000 Clermont-Ferrand, France. E-mail: sylvie.droitvolet@uca.fr 


\begin{abstract}
This study examined the judgment of the passage of time in elderly people living in retirement homes, focusing on the passage of time experienced in the present and that judged retrospectively for short periods (last day, week, month) and longer periods of life (year, now compared with 5 years before, as we get older). Participants' cognitive abilities and feeling of happiness were also assessed among other dimensions. Results showed no significant relationship between these three forms of judgment of the passage of time, except between the judgment of the passage of time for the present and for the day. In addition, the level of happiness was a significant predictor of both the momentary judgment of the passage of time and the retrospective judgment of the passage of time for shorter periods. In contrast, the individual differences in cognitive abilities better explained differences in the retrospective judgment of the passage of time for longer periods. As discussed, the different forms of judgments of the passage of time are therefore based on different cognitive mechanisms.
\end{abstract}

Keywords: Time; Passage of time; Aging; Consciousness; Happiness; Memory 


\section{INTRODUCTION}

How fast does time seem to pass for you? It makes no sense to ask people this question if we do not give them a time frame. Their answers can be based on their direct experience of the passage of time in the present moment or on comparisons between the rhythm of their lives in the present and in different past periods. And the underlying cognitive processes are likely to differ according to the type of judgment.

A distinction is made between two types of judgment of the passage of time: the retrospective passage-of-time judgment (retro-PoT) and the present passage-of-time judgment (present-PoT) (Wearden, 2015). The retro-PoT judgment is the judgment of the passage of time for an entire period of time that has already elapsed, i.e. in the past. The past period considered can be more or less long, covering a day, a week, a month or several years. The questions typically asked to participants are: How fast has time passed for you during the last week/month/year/5-10 years? The period considered can also cover an entire span of life as in the question "Do you think that time passes more quickly when one gets older? (e.g., Friedman \& Janssen, 2010; Janssen et al., 2013; Joubert, 1990; Lemlich, 1975; Wittmann \& Lehnoff, 2005; Wittmann et al., 2015).

The present-PoT judgment is the evaluation of the immediate sense of the speed of the passage of time, that is the momentary temporal judgment made in the present. It is closely linked to the context in which the judgment is made. To evaluate this type of temporal judgment, researchers have used Experienced Sampling Methodology (ESM), also called Ecological Momentary Assessment (EMA) (Droit-Volet, 2019; Droit-Volet \& Wearden, 2015, 2016; Droit-Volet et al., 2017). Indeed, this method permits the evaluation of the PoT judgment at different times of the day. In addition, each temporal question is associated with a series of other questions about the context (emotion, activity) in which the temporal judgment is made. Some researchers have also attempted to examine this temporal judgment by asking participants to answer one question on their current life: How fast does time usually pass for you? (Winckler et al., 2017; Janssen et al., 2013). However, this judgment is ambiguous because it is not clear whether participants base their judgments on direct experience of the PoT at time $t$, or on a period of several days, weeks or months, i.e. on life in general.

The few studies on PoT judgments have found different results depending on the type of temporal judgment requested. Although the data are not always consistent across studies (Droit-Volet \& Wearden, 2015), most of them have found an effect of age on the retro-PoT judgment when a long period of life was taken into account, i.e. for questions covering periods 
of 5 or 10 years and questions relating to changes in PoT with aging (e.g. Friedman \& Janssen et al., 2013; Lemlich, 1975; Winkler et al., 2017; Wittmann \& Lehnhoff, 2005). For these two long periods, the PoT was judged faster by older participants (older than 50 years) than younger participants. In contrast, for the retro-PoT judgment of shorter time periods (day, week, month), no age effect has been observed (Droit-Volet \& Wearden, 2015, 2016; Friedman \& Janssen, 2010; Janssen et al., 2013; Winkler et al., 2017; Wittmann \& Lehnhoff, 2005; Wittmann et al., 2015). Similarly, the ESM studies on the present-PoT judgment have not found any difference between older and younger participants (Droit-Volet \& Wearden, 2015, 2016; Droit-Volet et al., 2017). A recent ESM study conducted by Droit-Volet (2019) even showed a reversal in the daily feeling of the passage of time toward a slowing down of time in the participants older than 75 years of age, and especially those living in retirement homes. In recent studies of PoT during the COVID lockdown using a large sample, Ogden (2020) and Droit-Volet et al. (2020) also found a significantly slower passage of time with increasing age, alhough this age effect was not replicated in another COVID study (Martinelli et al., 2021). One can therefore have the experience that the years flash past, but that the days last forever (DroitVolet \& Wearden, 2015).

The differences in the results according to the type of temporal judgment can be explained by the different psychological mechanisms that underlie each type of judgment. ESM studies have found that the main predictor of the present-PoT judgment was not age but the affect felt at the moment of the judgment, namely the level of happiness felt (Droit-Volet \& Wearden, 2015, 2016; Droit-Volet et al., 2017; Droit-Volet, 2019). Participants have indeed reported that time passes faster when they are happy and drags when they are sad (less happy). The feeling expressed by seniors living in retirement homes that time slows down is therefore associated with a decrease in their level of happiness (Droit-Volet, 2019). Indeed, seniors living in retirement homes often present symptoms of depression or anxiety (Seitz et al., 2010). Other significant predictors of the present-PoT judgment have nevertheless been identified, namely the level of arousal, the degree of attention devoted to the current task, the feeling of frustration (Tipples, 2018), time pressure or the number of routines involved in daily life (Janssen et al., 2013; Winkler et al., 2017). The present-PoT judgment does indeed depend on the context in which it is made, and this varies according to the population tested and their lifestyle. However, as argued by Winkler et al. (2017), the two main predictors of judgment of current passage of time are emotion and attention.

The present-PoT judgment is therefore closely related to the emotional state experienced by participants in the present moment. The relationship between the emotion felt (happiness) 
and the other forms of PoT judgment has not yet been investigated. Nevertheless, we can assume that the feeling of happiness is less related to the retro-PoT judgment than to the presentPoT judgment, with the latter being based on emotions experienced in the present moment and the former on recovery processes in long-term memory (Friedman \& Janssen, 2010; Janssen, 2017). However, there may also be a difference due to the period of time considered, i.e. whether it is short (day, week, month, year) or long (5-years, PoT with aging). Indeed, retroPoT judgments for shorter periods (i.e., day, week, month, year) imply episodic memory, which is based on certain memories of personal events of daily life together with their emotional dimension (Di Crosta \& La Malva, 2017). On the other hand, retro-PoT judgments for longer periods seem to be based more on knowledge of self in its temporality (narrative self, Gallagher, 2000), detached from daily experiences. For example, most people think that time passes faster as they get older. But this thought does not correspond to any reality (Miller, 2019). In everyday life, time does not pass more quickly in older than in younger people (e.g., Droit-Volet \& Wearden, 2015). As Pierre Janet (1928) said many years ago, it is a kind of "collective illusion": "There is no contradiction between us, we all have the same language about this optical illusion" (p. 43). This idea in fact comes from knowledge of self in its temporality, i.e., from the deduction in relation to our age that we have little time left to live before the probable date of our death (Droit-Volet \& Dambrun, 2019). Therefore, the retro-PoT judgment for long periods would depend on processes related to semantic memory rather than episodic memory with its emotional coloring, although the two do not operate separately. In this case, it can be assumed that the inter-individual differences in the retro-PoT judgment for long periods may be related to individuals' cognitive abilities rather than to the feeling of happiness experienced in everyday life. In contrast, the retro-PoT judgment of shorter periods (day, week, month) may also be related to individuals' level of happiness.

The aim of this study was to examine the different types of judgment of the passage of time (retro-PoT, present-PoT) and the links between them, as well as their predictors: the level of happiness felt and/or the cognitive capacities. We conducted this study with old participants living in the same retirement home for three main reasons. The first was to find participants with different cognitive abilities in order to examine our hypotheses. The second was to avoid differences in temporal judgments related to the place of residence. The third was to try to replicate the results found by Droit-Volet (2019) in elderly persons at different stages of dementia. Participants' cognitive abilities were assessed using the MoCA, which is used to diagnose dementia (Montreal Cognitive Assessment) (Nasreddine et al., 2005). Participants' levels of depression and anxiety were also evaluated using the Beck Depression Inventory 
(BDI, Beck et al. 1961) and the State-Trait Anxiety Inventory (STAI, Spielberger et al., 1983), respectively. We used ESM for the present-PoT judgment and a series of questions for the retrospective-PoT judgment. Self-reported level of happiness was assessed after each time question for the former and after the series of questions for the latter.

\section{METHOD}

\section{Participants}

The final sample was composed of 53 elderly adults (40 women and 13 men) with a mean age of 87.5 years $(\mathrm{SD}=7.62, \mathrm{Min}=65, \mathrm{Max}=100)$. They were all living in the same retirement home in Cusset in the Auvergne Rhône-Alpes Region of France. They were recruited in cooperation with the clinical psychologist of the retirement home. The MoCA was used to estimate their cognitive impairment $\left(\mathrm{M}_{\mathrm{MoCA}}=16.06, \mathrm{SD}=5.77, \mathrm{Min}=4\right.$, $\left.\mathrm{Max}=29\right)$; lower scores indicating greater cognitive impairments. The MoCA scores allowed us to identify two groups of participants with significantly different levels of cognitive impairment: moderate cognitive impairments $\left(\mathrm{N}=28, \mathrm{M}_{\mathrm{MoCA}}=11.68, \mathrm{SD}=3.15\right.$, $\left.\mathrm{Min}=4, \mathrm{Max}=16\right)$, and mild or very mild cognitive impairments $\left(\mathrm{N}=25, \mathrm{M}_{\mathrm{MoCA}}=20.96, \mathrm{SD}=3.15, \operatorname{Min}=13, \operatorname{Max}=29\right)$, $F(1,51)=97.18, p<.0001$. Age and level of education were similar in these two groups (88 vs. 87 years, $F(1,51)=0.73, p=.40 ; 8.59$ vs. 8.8 years of education, $F(1,51)=0.24, p=.62$. All the participants volunteered to participate in this experiment and signed a consent form approved by the Sud-Est VI Statutory Ethics committee.

\section{Materials and Procedure}

For the retrospective PoT judgments (retro-PoT), the participants responded to a series of 7 questions on a 7-point Likert-type scale (from 1 "not at all - very slow" to "very much very fast"): "Do you find that time has passed quickly today (1), this week (2), this month (3), this year? (4)", ' 'Do you find that time passes more quickly now than 5 years ago (5), and that time is passing faster as you get older (6)?'. The retro-PoT for the day (7) was averaged over the responses given at the end of each of the five ESM testing days (between 7 and 8 p.m.). After the retro-PoT questions, the participants answered another question on their level of happiness, again with a 7-point response scale.

For the present PoT judgment (present-PoT), the ESM adapted for very old people was 
used (Droit-Volet, 2019). The participants had to spontaneously answer a temporal question on a 7-point scale (from 1 very slow to 7 very fast): "Now, at this moment, how fast does time seem to be passing for you.' They responded to this question twice a day for five consecutive weekdays (from Monday to Friday). The questions were asked by an experimenter. The questioning time had previously been fixed based on a random choice between 8 and 12 a.m. for the first question, and between 12.30 and $6.30 \mathrm{p} . \mathrm{m}$. for the second question. As in other ESM studies, after the temporal question, the participants answered the affective questions (happiness, high-arousal, low-arousal) and the attention-related questions (Is the activity that you are doing difficult? Does it capture your attention?). They also answered a final question about their level of pain, because focusing on their physical pain could change their relationship to time. They responded on a 5-point scale for the pain question and a 7-point scale for the other questions.

In addition to the MoCA (Nasreddine et al., 2005), symptoms of depression and anxiety were assessed using the BDI (Beck et al., 1961), and the STAI (Spielberger et al., 1983), respectively. These scales, as well as the MoCA and the retro-PoT questions, were administered two or three days before and after the ESM. An additional question was asked on the participants' judgment of their physical and psychological health: from very good to very bad. Participants' responses to the question on psychological health were not taken into account because this variable was confused with the feeling of being happy $(r=0.84, p<0.0001)$.

\section{Statistical analyses}

Different statistical analyses were conducted for the retrospective and the present PoT judgment. For the analyses on the retro-PoT judgment, we conduced a series of independent samples $t$-tests on this temporal judgment for each period considered (day averaged, week, month, year, 5 years, as you get older) to compare the groups of participants with moderate vs. mild/very mild cognitive impairments. In addition, we analyzed the correlations between the different retrospective time judgments and the scores for happiness, cognition (MoCa), depression and anxiety. Preliminary statistical analyses on the retro-PoT judgment showed no significant relationship between the self-reported level of physical health and the different judgments of the passage of time (all $p>.05$ ). This factor was thus excluded from the statistical analyses.

For the present-PoT judgment, there were multiple responses per participant for each testing trial. Therefore, we conducted regression analyses (Multi-Level Modeling, MLM) using 
SPSS with the present-PoT as dependent variable and the participants as random effect. Initial statistical analyses were conducted with the two repeated measures, i.e. the testing day (numbered from 1 to 5) and the moment of assessment (a.m. vs. p.m.), as fixed factors using the autoregressive covariance structure $(A R(1))$. The results showed neither an effect of the testing day $(\mathrm{E}=0.18, S E=0.11,95 \% \mathrm{CI}[-0.04 ; 0.40], t=1.64, p=.10)$, nor of the assessment moment $(\mathrm{E}=0.21, S E=0.24,95 \% \mathrm{CI}[-0.25 ; 0.66], t=0.90, p=.37)$, nor of their interaction $(\mathrm{E}=-0.09, S E=0.07,95 \% \mathrm{CI}[-0.22 ; 0.66], t=-1.22, p=.23)$. Therefore, these factors were excluded from the subsequent MLM analyses testing the fixed effects (cognition, depression, anxiety, happiness, high-arousal, low-arousal, activity difficulty, activity attention, pain) entered in the same model.

To examine the relationship between the present-PoT judgment and each type of retroPoT judgment, we also conducted a MLM analysis with the present PoT judgment as dependent variable and each type of retro-PoT judgment taken separately as fixed factor. The participants were always used as random factor.

Additional Bayesian analyses were conducted using JASP for the $t$-tests, the correlation matrix $\left(\mathrm{BF}_{10}\right.$, Upper 95\% CI, Lower 95\% CI), and regression analyses to verify whether with Bayes Factors indicated that the significant and the non-significant results supported the alternative $\left(\mathrm{H}_{1}\right)$ and the null hypothesis $\left(\mathrm{H}_{0}\right)$, respectively (Dienes, 2014).

\section{RESULTS}

\section{The retrospective judgment of the passage of time}

The correlation matrix between the retro-PoT judgments and the scores on the different scales is presented in Table 1. Table 1 suggests some differences in the retrospective time judgments according to the periods considered, i.e., between the shorter periods (day, week, month) and the longer periods (year, now than 5 years before, as one gets older).

As illustrated in Figure 1, the PoT judgment did not change with the cognitive impairment scores (MoCA scores) for the short periods (day, week, month) (for all $r, p>.05$ ), although there was nevertheless moderate support for $\mathrm{H}_{0}$. The $t$-tests confirmed the similarity in the temporal judgment between the participants with moderate and mild cognitive impairment for the day $\left(\mathrm{M}_{\text {mod. }}=4.92, \mathrm{SE}=0.02 ; \mathrm{M}_{\text {mild }}=5.08, \mathrm{SE}=0.02 ; t(51)=0.62, p=0.54\right.$; Cohen's $\left.\mathrm{d}=0.17 ; \mathrm{BF}_{10}=0.32\right)$, the week $\left(\mathrm{M}_{\text {mod. }}=4.70, \mathrm{SE}=0.22 ; \mathrm{M}_{\text {mild }}=4.96, \mathrm{SE}=0.23\right.$; 
$\left.t(51)=0.78, p=0.44 ; \mathrm{d}=0.22 ; \mathrm{BF}_{10}=0.36\right)$, and the month $\left(\mathrm{M}_{\text {mod. }}=4.75, \mathrm{SE}=0.23 ; \mathrm{M}_{\text {mild }}=\right.$ $\left.5.04, \mathrm{SE}=0.25, t(51)=0.85, p=.40 ; \mathrm{d}=0.22 ; \mathrm{BF}_{10}=0.37\right)$ (Figure 1). Bayes factors supported $\mathrm{H}_{0}$, albeit moderately. For these short periods, rather than individual differences in the cognitive abilities, it was the variations in the feeling of happiness that influenced the time judgment (day, $r=0.45, p=.001, B F_{10}=38.45,95 \% \mathrm{CI}[0.64,0.19]$; week, $r=0.34, p=.05, B F_{10}=3.41,95 \%$ CI[0.56, 0.07]; month, $\left.r=0.37, p=.01, B F_{10}=5.26,95 \% \mathrm{CI}[0.57,0.10]\right)$. Strong evidence for the correlation between the feeling of happiness and the PoT judgment was found for the day $\left(B F_{10}>30\right)$, and moderate evidence was found when the length of the period increased, i.e. for the week and the month $\left(B F_{10}>3\right)$. Therefore, the participants experienced a slowing-down of time when they felt less happy over different temporal periods ranging from one day to one month.

Unlike the time judgment for the short periods, the corresponding judgment for the longer periods (year, now-5 years, when people get older) was not significantly related to the feeling of happiness (Table 1 , for all $r, p>.05$ ), as confirmed by the moderate evidence in support of $\mathrm{H}_{0}$ indicated by the Bayes factors $(B F 10>3)$, at least for the longest time period ( 5 years, when people get older). It was more related to mental capacities, i.e. the cognitive scores on the MoCA (year, $r=0.27, p<.05, B F_{10}=1.14,95 \% \mathrm{CI}\left[0.49,6.234^{\mathrm{e}-4}\right]$; now-5 years, $r=$ $0.47, p>.001, B F_{10}=82.29,95 \%$ CI $[0.65,0.22]$; aging, $r=0.28, p<.05, B F_{10}=1.35,95 \%$ $\mathrm{CI}[0.50,0.01])$. As confirmed by the $t$-test (see Figure 1), the participants with moderate cognitive impairment reported that time passed slower than those with mild cognitive impairment both when considering the past year $\left(\mathrm{M}_{\bmod }=4.82, \mathrm{SE}_{\bmod }=0.19 ; \mathrm{M}_{\text {mild }}=5.40, \mathrm{SE}\right.$ $\left.=0.21, t(51)=2.04, p=0.046, \mathrm{~d}=0.56, B F_{10}=1.50\right)$, now versus 5 years before $\left(\mathrm{M}_{\text {mod }}=2.75\right.$, $\left.\mathrm{SE}_{\text {mod }}=0.28, \mathrm{M}_{\text {mild }}=4.6, \mathrm{SE}_{\text {mild }}=0.298, t(51)=4.51, p<0.001, \mathrm{~d}=1.24, B F_{10}=511.87\right)$ and when people get older $\left(\mathrm{M}_{\text {mod }}=2.75, \mathrm{SE}_{\bmod }=0.32, \mathrm{M}_{\text {mild }}=3.92, \mathrm{SE}_{\text {mild }}=6.36, t(51)=\right.$ $2.52, p=.015, \mathrm{~d}=0.694, B F_{10}=3.52$ ). However, the Bayes factor only provides evidence for a cognitive impairment effect on the retrospective PoT judgment over the longest periods (5 years, as we get older). The evidence in favor of $\mathrm{H}_{1}$ was anecdotal for the year, suggesting that this time period might be a transitory period between the short and the very long periods of time.

\section{The present judgment of the passage of time}


The MLM results (Table 2) indicate that the main factor that significantly predicted the present-PoT judgment was the level of happiness experienced by the participants at the moment of the time judgment $(\mathrm{E}=0.037, S E=0.05,95 \% \mathrm{CI}[0.27 ; 0.47], t=7.13, p=.0001)$. The Bayesian analysis with happiness as the only factor confirmed this significant result $(E=0.44$, $\mathrm{SE}=0.06,95 \%$ CI $[0.32 ; 0.56], R-h a t=1.00, E_{\text {SS }}$ bulk $\left.=3295.45, E S S_{\text {tail }}=4007.63\right)$. The attention devoted to the current activity also contributed to variation in PoT, albeit to a lesser extent $(\mathrm{E}=0.079, S E=0.036,95 \% \mathrm{CI}[0.09 ; 0.149], t=2.22, p=.03)$. This was verified in the Bayesian analysis with the current activity as the only factor $(\mathrm{E}=0.14, S E=.04,95 \% \mathrm{CI}[0.06$; 0.23], $R$-hat $\left.=1.00, E S S_{\text {bulk }}=3592.66, E S S_{\text {tail }}=4121.39\right)$. As illustrated by the plots of the regression curve (Figure 2), at each moment of the day when the participants felt less happy, they expressed the feeling that time slowed down. Conversely, they reported that time passed faster as their level of happiness increased.

As we will discuss later, the present-PoT judgment therefore fluctuated greatly in terms of the level of self-reported happiness over the days $(\mathrm{M}=4.4, \mathrm{SD}=1.31$, Min =1, Maxi =7). The current feeling of happiness thus often alternated from pleasure to displeasure, with the result that there was no clear link between daily levels of happiness and the individual trait of depression, and a mild link between happiness and anxiety as indicted by the Bayesian MLM analyses $\left(\mathrm{E}=0.04, S E=0.02,95 \% \mathrm{CI}[-0.09 ; 0.02], R-h a t=1.02, E S S_{\text {bulk }}=170.25, E S S_{\text {tail }}=\right.$ $215.31 ; \mathrm{E}=-0.03, S E=0.01,95 \% \mathrm{CI}[-0.05 ;-0.01], R$-hat $=1.00, E_{\text {S }}$ bulk $=12547, E S S_{\text {tail }}=$ 2084.68, respectively).

\section{The relationship between the retrospective and the present judgment of the passage of time}

The results of the regression analyses (MLM) on the present passage-of-time judgment show that the different retro-PoT judgments were not related to the present-PoT judgment (all $p>$.05) (Table 3). These non-significant results were verified in the Bayesian analysis (week, $\mathrm{E}=0.13, \mathrm{SE}=0.10,95 \% \mathrm{CI}[-0.08 ; 0.34]$, month, $\mathrm{E}=0.12, \mathrm{SE}=0.09,95 \% \mathrm{CI}[-0.06 ; 0.31]$, year, $\mathrm{E}=-0.02, \mathrm{SE}=0.11,95 \% \mathrm{CI}[-0.24 ; 0.196], 5$ years, $\mathrm{E}=-0.01, \mathrm{SE}=0.07,95 \% \mathrm{CI}[-0.14$; $0.13]$, get older $\mathrm{E}=-0.03, \mathrm{SE}=0.07,95 \% \mathrm{CI}[-0.16 ; 0.10])$. The only significant link was between the different momentary judgments of time and the temporal judgment reported retrospectively at the end of the day $(\mathrm{E}=0.41, S E=0.05,95 \% \mathrm{CI}[0.31 ; 0.51], t=8.29, p=$ 
$.0001)$. This was confirmed by the Bayesian analyses $(\mathrm{E}=0.41, \mathrm{SE}=0.06,95 \% \mathrm{CI}[0.28 ; 0.52]$, R-hat $\left.=1.00, E S S_{\text {bulk }}=3041.37, E S S_{\text {tail }}=3954.54\right)$.

\section{DISCUSSION}

The results of our study on PoT judgment suggest that there was no significant relationship between the retrospective-PoT judgment and the PoT judgment at different present moments. The present-PoT judgment was only related to the judgment of the speed of the passage of time made retrospectively for the entire day. This supports the idea that PoT judgments involve different mechanisms depending on the time period under consideration.

Our results on the present-PoT judgment in everyday life obtained with the ESM are entirely consistent with the findings of other studies using this method (Droit-Volet, 2019; Droit-Volet \& Wearden, 2015, 2016; Droit-Volet et al., 2017). They confirm that the main predictor of the present-PoT judgment is the emotion felt at the moment of the temporal judgment. The activity carried out also plays a role, but to a lesser extent, probably because of the very wide range of activities performed. The emotion driving the variations in the PoT judgment in our study was the feeling of happiness. Participants reported that time passed faster when they felt happier and slowed down when their level of happiness decreased. Consequently, as shown by Droit-Volet (2019), elderly people living in retirement homes, such as those tested in our study, experienced a daily slowing down of time that was greater than that of elderly people (same age) living at home due to their enjoyment of fewer moments of happiness. To improve their mental health (wellbeing), it is therefore important to try to make elderly people happier by restoring meaning to their lives and making them accept their agedrelated changes (loss of autonomy), as suggested by the Acceptance and Commitment Therapy (ACT) for the elderly (Davison et al., 2017). The feeling of happiness is therefore a key factor in the variations in the experience of the passage of time in everyday life.

The feeling of being happy from day to day (emotion-state) is thought to change with general mental health, i.e. whether or not participants suffer from depression or anxiety (emotion-trait). It is well known that depressed people have a sad mood and lose interest in daily activities (Rottenberg, 2005). However, our statistical analysis indicated that the PoT judgment varied little with the individual scores of depression and anxiety on the self-reported clinical scales used. Similarly, it did not depend on general cognitive capacities as assessed by the MoCA in our study. In their COVID study on time, Martinelli et al. (2021) also found few relationships between the individual traits (durable happiness, impulsivity, depression, anxiety, 
alexithymia) and the momentary judgment of the passage of time, with this latter depending on the current emotional state (i.e., boredom, happiness) as in our study. Therefore, the emotional state always fluctuates from one moment to the next in everyday life according to the current context, despite a general personality trait. individual traits. This provides evidence in support of the distinction between "fluctuating happiness" in "which phases of pleasure and displeasure alternate repeatedly" and "authentic-durable happiness" which is "less dependent on circumstances" (Dambrun et al., 2012, p. 1). This also reinforces the idea that present-PoT judgment is effectively rooted in a unique experience carried out in a specific context (Martinelli \& Droit-Volet, in press). Our idea (Droit-Volet, 2018, 2019; Droit-Volet \& Dambrun, 2019) that the judgment of the passage of time in daily life is derived from the minimal self (subject of experience) (Gallagher, 2000, 2013) and its temporally contraction and extension under the influence of the emotion experienced at a given moment is therefore confirmed in our study.

Our results identified the feeling of being more or less happy as the major predictor of variations in the present-PoT judgment. The recent studies on the PoT judgment during the COVID lockdown have confirmed the role of this critical emotion, but also found an effect of other feelings such as boredom and life satisfaction (Ogden, 2020; Droit-Volet et al. 2020; Martinelli et al., 2021). Although these factors likely fall into two main categories (affect and attention load), it will be interesting to examine the specificities of different dimensions (e.g., anger, fear, boredom, frustration) and their inter-relations in order to identify whether one category of factors prevails over the other in the momentary judgment of the passage of time. However, as discussed above, the factors underlying this type of temporal judgment depend on the context in which the judgment is made (Martinelli et al., 2021). In this respect, the life context differs greatly between young and very old people living in retirement home.

Our study also confirms the weak relationship between the present- and the retro-PoT judgment. This validates the hypothesis that the retro-PoT judgment is not based on short-term cognitive mechanisms involved in the current experience as for the present-PoT judgment, but on recollection processes in long-term memory. In addition, our study suggests some differences in the retro-PoT judgment depending on the time period considered. Indeed, there was no or little significant correlation between the temporal judgment for the shorter periods (day, week, month) and that for the longer periods (5-years, aging). The PoT judgment for the last year was at an intermediate point, being significantly correlated with both the temporal judgment for the shorter periods (week, months) and that for the longer periods (5-years, aging), except that for the day. The best predictor of variations in the PoT judgment also differed as a 
function of the temporal period considered, being the level of happiness for the shorter periods and the level of cognitive impairment, i.e. the MoCA scores, for the longer ones. This suggests that PoT judgment for periods covering the day, the week, but also the month, are based on memorable events of everyday life stored in episodic memory. However, since there was no significant relationship between the judgment of time in the present moment and that of the last week or month, we can easily assume that PoT judgments for the last week or the last month do not result from the sum either of PoT experiences at different moments or of PoT judgments at the end of each day of the week, but from certain remarkable and memorable days. The latter would provide an emotional color (feeling of happiness) to the elapsed temporal period (week, month), thus influencing in turn the retrospective judgment of the passage of time. Further experiments are nevertheless needed to better understand the interplay between the emotional judgment of different days of the week, and that of the week and of the month as a whole.

Our results showed that the retrospective judgment of the passage of time over longer periods (year, 5-years, aging) was significantly correlated with the MoCA scores, rather than with the retro-PoT judgment for shorter periods (day, week, month) or the feeling of happiness, which is a reliable predictor of this latter judgment. This result cannot be explained by the older participants' difficulties in understanding some of the temporal questions as no cognitive impairment effects were observed for most of the temporal questions, which were similarly worded. On the other hand, the existence of memory deficits in the early stages of dementia is well known. The differences in the retro-PoT judgment for the longer periods between people with mild and those with moderate dementia symptoms could therefore be explained by the latter's problems in remembering past events. Defending a memory-based theory of retro-PoT judgment, Fraisse (1963) explained that time is thought to pass quickly with aging because the number of events retrieved in memory is smaller in older people than in younger people. The number of memories would therefore be even lower in people with more severe symptoms of dementia such as those tested in our study. However, this explanation is valid for the feeling of an acceleration of time with aging but not its slowing-down as was observed in our participants. In addition, recent studies have questioned this memory theory and suggested that the speedingup of time with aging is rather related to the experience of time pressure in old people (Janssen, 2017; Friedman \& Janssen 2010; Winkler et al., 2017; Wittmann \& Lehnhoff, 2005). However, it is hard to imagine that older people have a greater sense of time pressure when most of them no longer have the pressure of a job and/or family to manage. Is it the real pressure of everyday life or the pressure of life going by? 
The difference in the PoT judgment for the longer periods between the groups of participants with moderate and mild dementia can be better explained by their different abilities to represent time. Indeed, people with dementia symptoms have trouble orienting themselves in time (Eslinger et al., 1985). They have difficulties saying what day, month, year it is. Moreover, the questions on time orientation form an integral part of the MoCA test. However, although this explanation is valid, it is nevertheless also insufficient to account for the stronger sense of slowing down of time in the participants with the most severe dementia symptoms. The problem with studies using elderly people living in retirement homes is the heterogeneity of the population used, which presents many different clinical problems. In our study, it would have been preferable to have a larger number of participants in order to obtain very strong rather than moderately significant effects like those found in our study and to permit the addition of an age-matched control group without cognitive difficulties.

Our results indicate that the judgment of the passage of time for long periods of time was related neither to the participants' current emotion, nor to pain, nor to their self-reported level of physical health. We can therefore simply assume that this subjective retrospective judgment of time for very long periods of life is rooted in the awareness of ineluctable changes in the self with aging. Participants would thus compare the pace of their lives before and after their psychological difficulties, thus resulting in the statement that the pace of life has slowed down. The retro-PoT judgment for long periods would thus be linked to the concept of self in its temporality. The knowledge of self in its temporality (past, present, future) is linked to the narrative self (Gallagher, 2000, 2013).

Therefore, based on our results, we assume that both the representation of time and the concept of self take account of changes in the judgment of the passage of time for long periods (5-years, aging). Time representation and self-knowledge form part of the semantic memory system. This therefore supports our idea that the PoT-judgment for long periods of life involves semantic memory processes rather than episodic memory processes, as is the case for the time judgment of shorter periods (day, week, month). For future research, it therefore seems to be very important to evaluate participants' memory abilities by means of specific semantic memory tests and not only the MoCA, which rapidly evaluates cognitive function with different short subtests (visuo-spatial executive function, clock, time orientation, naming, short-term memory, attention, language, abstraction, memory recall) but not the cause of cognitive impairments. However, as already reported, it is obvious that episodic memory and semantic memory do not operate separately, as suggested by the findings showing the significant 
correlations between the PoT judgment for the last year and those for both the shorter and the longer periods.

In conclusion, our study has demonstrated that there is not one but several types of judgment of the passage of time depending on the temporal period taken as a reference and that these different time judgments are not or few related. This highlights the complexity of the PoT judgment in human consciousness (Gruber et al., 2018). Finally, talking about time would be a familiar way to account for ourselves, our moods and the way our difficulties increase with age. The slowing down of time in very old people would be a way of accounting for "things shipwrecked in the depths of the soul" in old age before death (Hugo, 1838).

\section{REFERENCES}

Beck, A. T., Ward, C., \& Mendelson, M. (1961). Beck depression inventory (BDI). Archives of General Psychiatry, 4, 561-571.

Dambrun, M., Ricard, M., Després, G., Drelon, E., Gibelin, E., Gibelin, M., Loubeyre, M., Py, D., Delpy, A., Garibbo, C., Bray, E., Lac, G., \& Michaux, O. (2012). Measuring happiness: from fluctuating happiness to authentic-durable happiness. Frontiers in Psychology, 3, 16. https://doi: 10.3389/fpsyg.2012.00016

Davison, T. E., Eppingstall, B., Runci, S., \& O’Connor, D. W. (2017). A pilot trial of acceptance and commitment therapy for symptoms of depression and anxiety in older adults residing in long-term care facilities. Aging \& Mental Health, 21(7), 766-773.

Dienes, Z. (2014). Using Bayes to get the most out of non-significant results. Frontiers in Psychology, 5, 781. https://doi: 10.3389/fpsyg.2014.00781.

Di Crosta, A., \& La Malva, P. (2017). Commentary: Experience Sampling Methodology reveals similarities in the experience of passage of time in young and elderly adults. Frontiers in Psychology, 8: 1384. doi: 10.3389/fpsyg.2017.01384

Droit-Volet, S. (2019). Time does not fly but slow down in old age. Time \& Society, O(0), 123.

Droit-Volet, S. (2018). Intertwined facets of subjective time. Current Directions in Psychological Science, 27, 422-428.

Droit-Volet, S. (2019). The temporal dynamic of emotional effect on judgments of durations. In V. Arstila, A. Bardon, S. Power \& A. Vatakis (dir.), The Illusions of Time: Philosophical and Psychological Essays on Timing and Time Perception (pp.103-125). Palgrave Macmillan, Springer Nature Switzeland A. 
Droit-Volet, S., \& Dambrun, M. (2019). Awareness of the passage of time and selfconsciousness: what do meditators report? PsyCH Journal, 8, 51-65. doi: 10.1002/pchj. 270

Droit-Volet, S., Gil, S., Martinelli, N., Andant, N., Clinchamps, M., Parreira, L., et al. (2020). Time and Covid-19 stress in the lockdown situation: Time free, «Dying» of boredom and sadness. PLoS ONE, 15(8): e0236465. doi.org/10.1371/journal.pone.0236465

Droit-Volet, S., Monceau, S. M., Dambrun, M., \& N. Martinelli, N. (2020). Embodied time and the out-of-body experience of the self. PeerJ 8: e8565. doi.org/10.7717/peerj.8565

Droit-Volet, S., \& Wearden, J. (2015). Experience Sampling Methodology reveals similarities in the experience of passage of time in young and elderly adults. Acta Psychologica, 156, 77-82.

Droit-Volet, S., \& Wearden, J. (2016). Passage of time judgments are not duration judgments: Evidence from a study using Experience Sampling Methodology. Frontiers in Psychology, 7, 176. doi: 10.3389/fpsyg.2016.00176.

Droit-Volet, S., Trahanias, P., \& Maniadakis, M. (2017). Passage of time judgments in everyday life are not related to duration judgments except for long durations of several minutes. Acta Psychologica, 73, 116-121.

Eslinger, P. J., Damasio, A. R., Benton, A. L., \& Van Allen, M. (1985). Neuropsychologic detection of abnormal mental decline in older persons. Journal of the American Medical Association, 253, 670-674.

Fraisse, P. (1963). The psychology of time. Harper and Row.

Friedman, W. J., \& Janssen, S. M. (2010). Aging and the speed of time. Acta Psychologica, 134, 130-141.

Gallagher, S. (2000). Philosophical conceptions of the self: implications for cognitive science. Trends in Cognitive Sciences, 4, 14-21.

Gallagher, S. (2013). A pattern theory of self. Frontiers in Human Neurosciences, 7: 443. https://doi: 10.3389/fnhum.2013.00443

Gruber, R. P., Smith, R. P., \& Block, R. A. (2018). The illusory flow and passage of time within consciousness: A multidisciplinary analysis. Timing and Time Perception, 6, 125-153.

Hugo, V. (1838). Ruy Blas. Fernand Nathan.

Janet, P. (1928). L'évolution de la mémoire et de la notion de temps. L'Harmattan.

Janssen, S. M. J. (2017). Autobiographical memory and the subjective experience of time. Timing \& Time Perception, 5, 99-122. 
Janssen, S. M., Naka, M., \& Friedman, W. J. (2013). Why does life appear to speed up as people get older? Time \& Society, 22, 274-290.

Joubert, C. E. (1983). Subjective acceleration of time: Death anxiety and sex differences. Perceptual and Motor Skills, 57, 49-50.

Lemlich, R. (1975). Subjective acceleration of time with aging. Perceptual and Motor Skills, 41, 235-238.

Martinelli, N., \& Droit-Volet, S. (In press). What factors underlie our experience of the passage of time? Theoretical consequences. Psychological Research.

Martinelli, N., Gil, S., Belletier, C., Chevalère, J., Dezecache, G., Huguet, P., \& Droit-Volet, S. (2021). Time and Emotion in the lockdown for the Covid-19 epidemic: The determinants of our experience of time? Frontiers in Psychology, 11: 616160. https://doi: 10.3389/fpsyg.2020.616169

Miller, K. (2019). Does it really seem to us as though time passes? In A. Valtteri, A. Bardon, S. E. Power, \& A. Vatakis (dir.), The illusions of time: Philosophical and Psychological Essays on Timing and Time Perception (pp. 17-35). Palgrave Macmillan, Springer Nature Switzeland A.

Nasreddine, Z. S., Phillips, N. A., Bédirian, V., Charbonneau, S., Whitehead, V., et al. (2005). The Montreal Cognitive Assessment, MoCA: a brief screening tool for mild cognitive impairment. Journal of the American Geriatrics Society, 53(4), 695-699.

Ogden, R. S. (2020). The passage of time during the UK Covid-19 lockdown. PLoS ONE, 15(7): e0235871. doi.org/10.1371/journal.pone.0235871

Rottenberg, J. (2005). Mood and Emotion in Major Depression? Current Direction in Psychological Science, 14(3), 167-170.

Seitz, D., Purandare, N., \& Conn, D. (2010). Prevalence of psychiatric disorders among older adults in long-term care homes: A systematic review. International Psychogeriatrics, 22, 10251039. doi: 10.1017/S1041610210000608

Spielberger, C. D., Gorsuch, R. L., Lushene, R., Vagg, P. R., \& Jacobs, G. A. (1983). Manual for the State-Trait Anxiety Inventory. Consulting Psychologists Press.

Tipples, J. (2018). Increased frustration predicts the experience of time slowing-down: Evidence from an experience sampling study. Timing \& Time Perception, 6, 220-230.

Wearden, J. H. (2015). Passage of time judgements. Consciousness and Cognition, 38, 165168.

Winkler, I., Fischer, K., Kliesow, K, Rudolph, T., Thiel, C., \& Sedlmeier, P. (2017). Has it 
really Been that Long? Why Time Seems to Speed up with Age. Timing \& Time Perception, 5, 168-189.

Wittmann, M., Rudolph, T., Gutierrez D., \& Winkler, I. (2015). Time Perspective and Emotion Regulation as Predictors of Age-Related Subjective Passage of Time. Int. J. Environ. Res. Public Health, 12(12), 16027-16042.

Wittmann, M., \& Lehnhoff, S. (2005). Age effects in perception of time. Psychol. Rep., 97(3), 921-935.

\section{Figure captions}

Figure 1. Mean (standard error) retrospective judgment of the passage of time from 1 (very slow) to 7 (very fast) made by the old participants with moderate cognitive impairment and those with mild or very mild cognitive impairments for the temporal questions on the day (averaged on the responses given at the end of 5 testing days), the week, the month, the year, now than 5 years ago, and as you get older.

Figure 2. Plot of the regression (MLM) between the present judgment of the passage of time from 1 (very slow) to 7 (very fast) and the level of self-reported happiness (standardized) assessed using the Experience Sampling Methodology.

\section{Table 1.}

Correlation matrix, including Pearson's $r$, the Bayesian factor $\left(B F_{10}\right)$, the Upper and the Lower 95\% Confident Interval $(C I)$, between retrospective judgments of the passage of time for different time periods (day, week, month, year, now compared to 5 years before, as people get older) and scores of happiness, cognition (MoCA), depression and anxiety.

\begin{tabular}{|c|c|c|c|c|c|c|c|c|c|c|}
\hline Variable & & $\mathrm{D}$ & $\mathrm{W}$ & $\mathrm{M}$ & $\mathrm{Y}$ & $5-y$ & $\mathrm{~A}$ & $\mathrm{H}$ & $\mathrm{C}$ & $\mathrm{D}$ \\
\hline \multirow[t]{4}{*}{ Week } & $r$ & 0.24 & - & & & & & & & \\
\hline & $B F_{10}$ & 0.70 & - & & & & & & & \\
\hline & $U 95 \% C I$ & 0.47 & - & & & & & & & \\
\hline & $L 95 \% C I$ & -0.04 & - & & & & & & & \\
\hline \multirow[t]{4}{*}{ Month } & $r$ & $0.33^{*}$ & $0.81 * * *$ & - & & & & & & \\
\hline & $B F_{10}$ & 2.64 & $2.683^{\mathrm{e}+10}$ & - & & & & & & \\
\hline & $U 95 \% C I$ & 0.54 & 0.88 & - & & & & & & \\
\hline & $L 95 \% C I$ & 0.06 & 0.67 & - & & & & & & \\
\hline \multirow[t]{4}{*}{ Year } & $r$ & 0.25 & $0.54 * * *$ & $0.70 * * *$ & - & & & & & \\
\hline & $B F_{10}$ & 0.82 & 777.40 & $2.297^{e+6}$ & - & & & & & \\
\hline & $U 95 \% C I$ & 0.48 & 0.70 & 0.81 & - & & & & & \\
\hline & $L 95 \% C I$ & -0.02 & 0.30 & 0.51 & - & & & & & \\
\hline \multirow[t]{2}{*}{5 -years } & $r$ & 0.10 & 0.25 & 0.16 & $0.31 *$ & - & & & & \\
\hline & $B F_{10}$ & 0.22 & 0.81 & 0.32 & 2.07 & - & & & & \\
\hline
\end{tabular}




\begin{tabular}{|c|c|c|c|c|c|c|c|c|c|c|}
\hline & $U 95 \% C I$ & 0.35 & 0.48 & 0.40 & 0.53 & - & & & & \\
\hline & $L 95 \% C I$ & -0.17 & -0.03 & -0.11 & 0.04 & - & & & & \\
\hline \multirow[t]{4}{*}{ Aging } & $r$ & 0.08 & 0.23 & $0.39 * *$ & $0.43 * *$ & $0.52 * * *$ & - & & & \\
\hline & $B F_{10}$ & 0.20 & 0.62 & 10.80 & 22.66 & 434.95 & - & & & \\
\hline & $U 95 \% C I$ & 0.33 & 0.46 & 0.59 & 0.61 & 0.69 & - & & & \\
\hline & $L 95 \% C I$ & -0.19 & -0.05 & 0.13 & 0.17 & 0.28 & - & & & \\
\hline \multirow[t]{4}{*}{ Happiness } & $r$ & $0.45 * * *$ & $0.34^{*}$ & $0.37 * *$ & 0.21 & 0.01 & -0.03 & - & & \\
\hline & $B F_{10}$ & 38.45 & 3.41 & 5.26 & 0.52 & 0.18 & 0.18 & - & & \\
\hline & $U 95 \% C I$ & 0.64 & 0.56 & 0.57 & 0.45 & 0.28 & 0.24 & - & & \\
\hline & $L 95 \% C I$ & 0.19 & 0.07 & 0.10 & -0.07 & -0.26 & -0.30 & - & & \\
\hline \multirow[t]{4}{*}{ Cognition } & $r$ & 0.12 & 0.21 & 0.13 & $0.27 *$ & $0.47 * * *$ & $0.28 *$ & -0.21 & - & \\
\hline & $B F_{10}$ & 0.24 & 0.50 & 0.26 & 1.14 & 82.29 & 1.35 & 0.50 & - & \\
\hline & $U 95 \% C I$ & 0.37 & 0.44 & 0.38 & 0.49 & 0.65 & 0.50 & 0.07 & - & \\
\hline & $L 95 \% C I$ & -0.15 & -0.07 & -0.14 & $6.234^{\mathrm{e}-4}$ & 0.22 & 0.01 & -0.45 & - & \\
\hline \multirow[t]{4}{*}{ Depression } & $r$ & -0.17 & -0.14 & $-0.28 *$ & -0.17 & 0.14 & 0.04 & -0.23 & 0.04 & - \\
\hline & $B F_{10}$ & 0.36 & 0.29 & 1.18 & 0.35 & 0.29 & 0.18 & 0.61 & 0.18 & - \\
\hline & $U 95 \% C I$ & 0.10 & 0.13 & -0.01 & 0.11 & 0.39 & 0.30 & 0.05 & 0.31 & - \\
\hline & $L 95 \% C I$ & -0.42 & -0.39 & -0.50 & -0.42 & -0.13 & -0.23 & -0.46 & -0.23 & - \\
\hline \multirow[t]{4}{*}{ Anxiety } & $r$ & -0.13 & -0.17 & -0.27 & -0.08 & 0.10 & 0.03 & -0.25 & -0.01 & $0.68 * * *$ \\
\hline & $B F_{10}$ & 0.27 & 0.34 & 1.01 & 0.20 & 0.22 & 0.18 & 0.81 & 0.18 & 37941530 \\
\hline & $U 95 \% C I$ & 0.14 & 0.11 & 0.010 & 0.20 & 0.35 & 0.29 & 0.03 & 0.26 & 0.80 \\
\hline & $L 95 \% C I$ & -0.30 & -0.41 & -0.49 & -0.33 & -0.18 & -0.24 & -0.48 & -0.28 & 0.48 \\
\hline
\end{tabular}

Note. Pearson'r; Bayes Factor; Upper 95\% CI; Lower 95\% CI; * $p<.05 ; * * p<.01 ; * * * p<.001$ 
Table 2. Results of the regression analysis (MLM) on the present passage-of-time judgments with the different factors entered in the same model.

\begin{tabular}{lcccccc}
\hline & & \multicolumn{6}{c}{$95 \%$ CI } \\
& Estimate $^{\mathrm{a}}$ & $\mathrm{SE}$ & Lower & Upper & $t$ & $p$ \\
\hline Cognition & 0.030 & 0.017 & -0.004 & 0.063 & 1.77 & 0.08 \\
Depression & -0.008 & 0.019 & -0.046 & 0.029 & -0.45 & 0.66 \\
Anxiety & 0.002 & 0.011 & -0.019 & 0.023 & 0.21 & 0.83 \\
Happiness & 0.368 & 0.052 & 0.267 & 0.470 & 7.13 & 0.0001 \\
High-arousal & -0.057 & 0.035 & -0.127 & 0.012 & -1.62 & 0.11 \\
Low-arousal & 0.010 & 0.046 & -0.079 & 0.100 & 0.23 & 0.82 \\
Activity difficulty & -0.051 & 0.036 & -0.122 & 0.020 & -1.42 & 0.16 \\
Activity attention & 0.079 & 0.036 & 0.009 & 0.149 & 2.22 & 0.03 \\
Pain & -0.091 & 0.053 & -0.195 & 0.013 & -1.72 & 0.09 \\
\hline
\end{tabular}

a Dependent variable: Present-PoT judgment 
Table 3. Results of the regression analyses (MLM) on the present passage-of-time judgment with each type of retrospective passage-of-time judgment considered as factor taken separately.

\begin{tabular}{lcccccc}
\hline & \multicolumn{6}{c}{$95 \%$ CI } \\
& Estimate $^{\mathrm{a}}$ & SE & Lower & Upper & $t$ & $p$ \\
\hline Day & 0.4081 & 0.0492 & 0.3112 & 0.5049 & 8.29 & 0.0001 \\
Week & 0.1291 & 0.1010 & -0.0736 & 0.3317 & 1.28 & 0.21 \\
Month & 0.1207 & 0.0931 & -0.0662 & 0.3075 & 1.30 & 0.20 \\
Year & -0.0158 & 0.1101 & -0.2368 & 0.2052 & -0.14 & 0.89 \\
5-years & -0.0025 & 0.0669 & -0.1368 & 0.1319 & -0.04 & 0.97 \\
Aging & -0.0277 & 0.0658 & -0.1599 & 0.1044 & -0.42 & 0.68 \\
\hline
\end{tabular}

a Dependent variable: Present-PoT judgment 
Figure 1

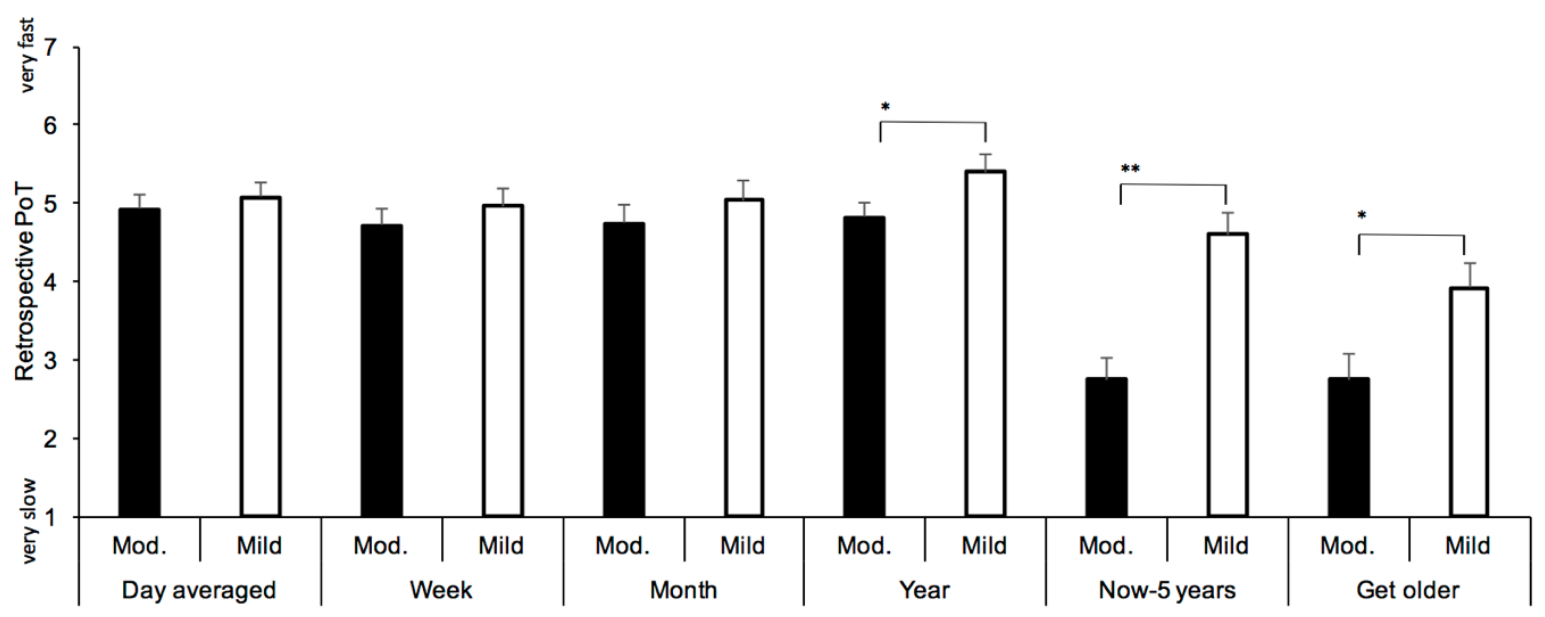


Figure 2

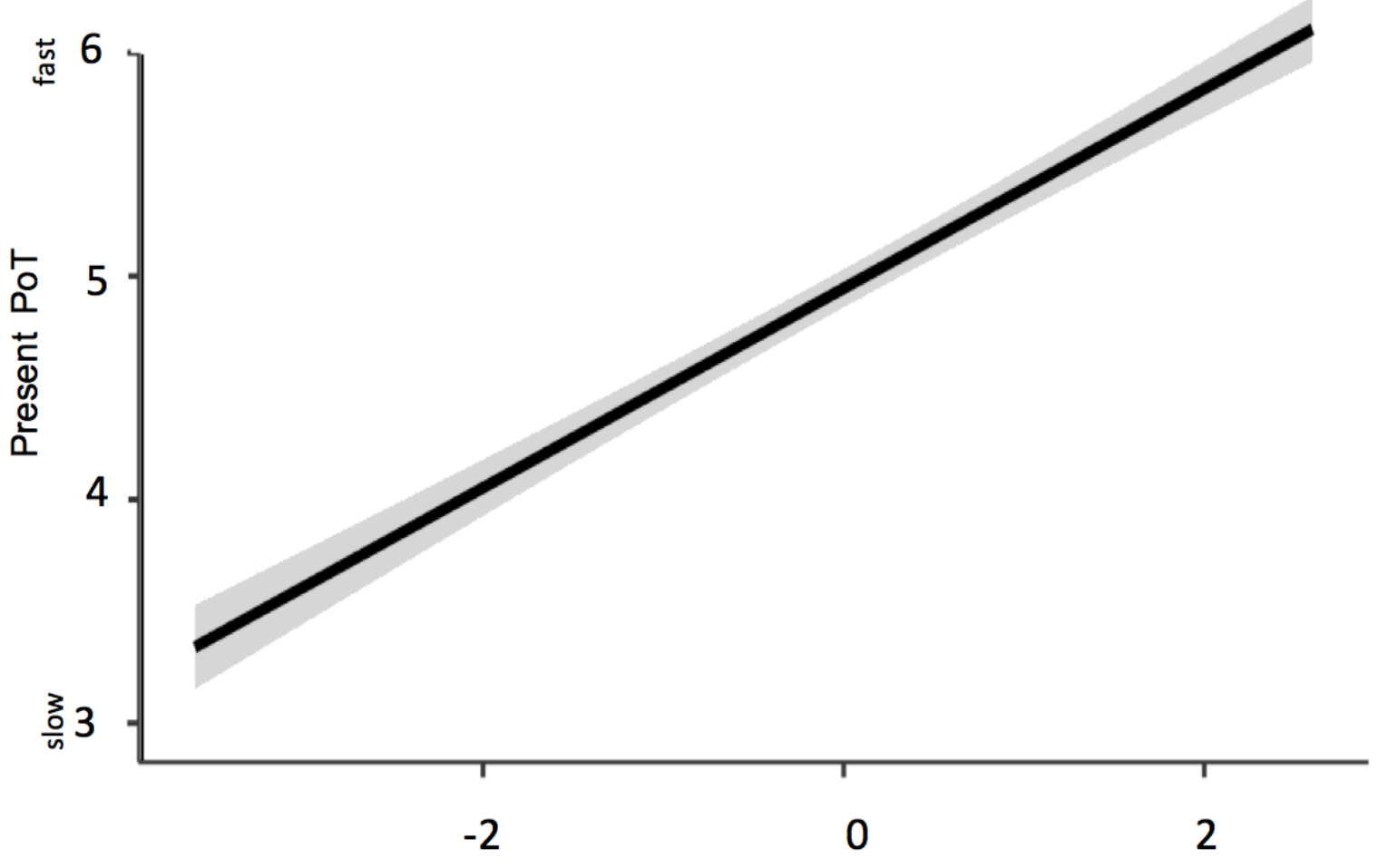

Happiness 